\title{
Mapeamento de recursos de tecnologia assistiva utilizados por idosos
}

\section{Mapping of assistive technology resources used by the aged}

\author{
Luma Carolina Câmara Gradim ${ }^{1}$, Shamyr Sulyvan de Castro ${ }^{2}$, \\ Darlene Mara dos Santos Tavares ${ }^{3}$, Alessandra Cavalcanti ${ }^{4}$
}

http://dx.doi.org/10.11606/issn.2238-6149.v27i1p72-79

Gradim LCC, Castro SS, Tavares DMS, Cavalcanti. Mapeamento de recursos de tecnologia assistiva utilizados por idosos. Rev Ter Ocup Univ São Paulo. 2016 jan.-abr.;27(1):72-9.

RESUMO: O aumento da expectativa de vida populacional no Brasil evidencia também o número de pessoas que envelhecem apresentando perda da capacidade funcional e aumento da dependência nas atividades do dia a dia. A tecnologia assistiva (TA) se constitui como suporte para que os idosos mantenham a independência e funcionalidade. Os objetivos deste estudo foram: (i) elencar os recursos de tecnologia assistiva utilizados por idosos assistidos em uma unidade matricial de saúde(UMS) no município de Uberaba/MG; (ii) descrever as características sócio-econômicas e as doenças crônicas por eles relatadas e (iii) estudar a área de ocupação - atividades de vida diária e a categoria dos recursos da tecnologia assistiva usados. Participaram do estudo 144 idosos com 60 anos ou mais. Para a coleta dos dados foram aplicados dois instrumentos estruturados idealizados pelos autores. Os resultados evidenciaram utilização de tecnologia assistiva aquém das necessidades para manutenção do desempenho funcional dos idosos, com exceção do uso de óculos. Aidade média dos participantes foi de 72,4 anos $( \pm 7,8)$ e houve predomínio de idosos do gênero feminino. Os diagnósticos clínicos relatados foram na maioria casos de hipertensão arterial. Dos participantes, $140(97,2 \%)$ relataram utilizar um tipo de recurso de TA como auxilio em alguma atividade de vida diária.

DESCRITORES: Equipamentos de autoajuda; Atenção primária à saúde; Terapia ocupacional; Idoso; Atividades cotidianas.
Gradim LCC, Castro SS, Tavares DMS, Cavalcanti. Mapping of assistive technology resources used by the aged. Rev Ter Ocup Univ São Paulo. 2016 Jan.-Apr.;27(1):72-9.

\begin{abstract}
The increase in life expectancy of the Brazilian population shows the number of people that age with functional capacity loss and increased dependency to perform daily activities. Assistive technology (AT) is a support for older adults to maintain their independence and functionality. The objectives of this study were: (i) to list assistive technology resources used by older adults assisted in a Centralized Health Unit (CHU) in the city of Uberaba, Minas Gerais, Brazil; (ii) to describe the socioeconomic characteristics and chronic diseases reported by them, and (iii) to study the area of occupation - daily life activities and the category of resources used. One hundred and forty-four older adults aged 60 years or more participated in the study. For the data collection, two structured instruments devised by the authors were applied. The results showed that the use of assistive technology was beneath the expected for maintenance of the functional performance of older adults, except for the use of glasses. The average age of participants was 72.4 years $( \pm 7.8)$ and there was a predominance of women. Reported diagnoses were, in most cases, hypertension. Of the participants, 140 (97.2\%) reported using a type of AT resource as an aid in some daily activity.
\end{abstract}

KEYWORDS: Self-help devices; Primary health care; Occupational therapy; Aged; Daily living activities.

Artigo decorrente do Trabalho de Conclusão de Curso (TCC) do Curso de Terapia Ocupacional da Universidade Federal do Triângulo Mineiro (UFTM) - aprovado pelo Comitê de Ética da UFTM de acordo com o parecer $n^{\circ}$ 2315/2012

1. Terapeuta Ocupacional graduada pela Universidade Federal do Triângulo Mineiro. Email: luma.carol90@hotmail.com

2. Professor Adjunto do Departamento de Fisioterapia Aplicada. Curso de Fisioterapia. Instituto de Ciências da Saúde. Universidade Federal do Triângulo Mineiro. Email: shamyr@fisioterapia.uftm.edu.br

3. Professora Associada do Departamento de Enfermagem em Educação e Saúde Coletiva. Curso de Enfermagem. Instituto de Ciências da Saúde. Universidade Federal do Triângulo Mineiro. Email: darlenetavares@enfermagem.uftm.edu.br

4. Professora Adjunta do Departamento de Terapia Ocupacional. Curso de Terapia Ocupacional. Instituto de Ciências da Saúde. Universidade Federal do Triângulo Mineiro. Email: alessandra@to.uftm.edu.br

Endereço para correspondência: Universidade Federal do Triângulo Mineiro. Unidade Centro Educacional (CE). Departamento de Terapia Ocupacional. Av. Getúlio Guaritá, nº 159. Térreo, sala T21. Uberaba, MG. CEP: 38025-440. 


\section{INTRODUÇÃO}

$\mathrm{O}$ envelhecimento populacional gera mudança demográfica e tem implicações para as políticas públicas de saúde. $\mathrm{O}$ aumento dessa população representa acréscimo da demanda para os serviços, em decorrência da probabilidade para aparecimento de doenças crônicas. No Brasil o número de pessoas idosas, com 60 anos ou mais, no ano de 2000, era de 14.536 .029 $(8,6 \%)$, e em 2010 foi de $19.321 .986(10,13 \%)^{1}$.

A fase do envelhecimento pode cursar com aumento da perda de papéis ocupacionais, alteração da funcionalidade, mudanças na organização da vida cotidiana e nos hábitos pessoais e, geralmente, é associada ao prolongado uso dos serviços de saúde 2 . Ainda, o idoso tende a ter dependência para a realização de suas tarefas cotidianas, que pode aumentar em aproximadamente 5\% aos 60 anos e $50 \%$ aos 90 anos ou mais ${ }^{3}$.

$\mathrm{Na}$ maioria das vezes, a capacidade funcional, está relacionada às habilidades presentes e à independência para a realização das atividades do dia a dia ${ }^{4}$. Ao passo que a incapacidade funcional pode ser aferida por meio do relato da dificuldade de desempenhar a tarefa ou atividade desejada ou pela evidência da necessidade de auxílio para realizá-las. No Brasil, de acordo com Giacomin et al. ${ }^{5}$, a incapacidade funcional pode atingir cerca de $2 \%$ a $47 \%$ dos idosos. A esse crescimento soma-se o baixo nível socioeconômico e educacional, e a alta prevalência de doenças crônicas causadoras das limitações funcionais e de incapacidades.

A fim de subsidiar ações que auxiliem o cenário, o Ministério da Saúde, disponibiliza produtos de tecnologia assistiva (TA) com a intenção de facilitar a realização de atividades cotidianas e promover independência funcional ${ }^{6,7}$. Esses produtos são elencados na lista oferecida pelo Sistema Único de Saúde (SUS), composta por itens como: cadeira de rodas, adaptador postural para cadeira de rodas, órtese e prótese ortopédica, além do serviço de Avaliação de Tecnologia em Saúde (ATS) utilizado no período de duração do produto. Este serviço deve acompanhar desde a etapa do desenvolvimento e gestão de uso nos serviços de saúde até a substituição ou obsolescência da tecnologia assistiva ${ }^{8}$.

A tecnologia assistiva é definida como uma área de conhecimento de diferentes profissionais da saúde e de outras áreas como a das engenharias, e reúne "produtos, recursos, metodologias, estratégias, práticas e serviços que objetivam promover a funcionalidade, relacionada à atividade e participação, de pessoas com deficiência, incapacidades ou mobilidade reduzida [...]" (p.10)7.
Segundo Garcia e Galvão Filho ${ }^{9}$, na última pesquisa nacional de tecnologia assistiva junto a instituições (ensino superior, empresas e entidades do terceiro setor) referentes aos anos de 2005 a 2008, os idosos e as pessoas com deficiência visual foram apontados como as populações que mais tiveram projetos de tecnologia assistiva dispensados $(35,8 \%)$; perdendo apenas para as pessoas com deficiência física $(43,1 \%)$.

Esta pesquisa espera ampliar a compreensão sobre o cenário que envolve o uso de dispositivos de tecnologia assistiva por idosos e possibilitar a análise prática dos recursos dispensados pelos serviços de saúde para essa população. Espera-se também trazer informações sobre o tema para as pessoas que trabalham (profissionais de saúde) e para as que buscam os serviços do Sistema Único de Saúde (SUS), acerca dos dispositivos de tecnologia assistiva (TA).

Assim, o estudo teve como objetivos (i) elencar os recursos de tecnologia assistiva em uso por idosos que utilizam os serviços de uma unidade matricial de saúde (UMS) no município de Uberaba/MG; (ii) descrever as características sócio-econômicas e as doenças crônicas por eles relatadas e (iii) estudar a área de ocupação atividades de vida diária e a categoria dos recursos da tecnologia assistiva usados.

\section{PROCEDIMENTOS METODOLÓGICOS}

Estudo de abordagem quantitativa com delineamento transversal, observacional e descritivo. O projeto foi aprovado pelo Comitê de Ética em Pesquisa com Seres Humanos da Universidade Federal do Triângulo Mineiro (CEP/UFTM), parecer $\mathrm{n}^{\circ} 2315$ de 2012. Todos os participantes assinaram o Termo de Consentimento Livre e Esclarecido, no momento da coleta de dados.

Pesquisa foi realizada em uma Unidade Matricial de Saúde (UMS), situada em um Distrito Sanitário de Saúde do município de Uberaba/MG. O mapa territorial da UMS é dividido em nove microáreas para o atendimento da população adstrita em serviços de especialidades básicas; servindo também de apoio às Unidades de Saúde da Família (USF's) e do Núcleo de Apoio à Saúde da Família (NASF).

A equipe está constituída por um clínico geral da rede e dois do Programa de Saúde da Família, além de dois ginecologistas, dois pediatras, um dentista, dois enfermeiros, três auxiliares de enfermagem, um psicólogo, um assistente social, um fisioterapeuta e três agentes comunitários (ACS). Também possui convênio com uma Instituição de Ensino Superior para estágio em nutrição e fisioterapia. 
Participaram do estudo, idosos com 60 anos ou mais, de ambos os gêneros, capazes de entender e responder as perguntas dos formulários, independente da sua condição de saúde, e cadastrados na UMS. Foram excluídos os idosos que não foram encontrados após três visitas do entrevistador; que mudaram de endereço, que foram a óbito ou recusaram a participar do estudo.

Para o cálculo amostral procedeu-se a análise de todas as fichas das famílias cadastradas na UMS. Selecionaram-se as pessoas que possuíam 60 anos ou mais de idade, totalizando 573 pessoas. Após essa seleção, foram excluídas aquelas que não estavam com identificação no sistema eletrônico do SUS, isto é, aquelas que não tinham os dados necessários para localização do domicílio, chegando a 513 idosos.

Para a amostra probabilística, determinou-se o tamanho da amostra $(n)$ de forma que todas as pessoas tivessem a mesma probabilidade para serem selecionadas utilizando a fórmula abaixo como cálculo amostral.

$$
n=\frac{(\mathrm{P} \times 1-\mathrm{p})}{\mathrm{e}_{\mathrm{a}}}
$$

Na qual: $n=$ tamanho da amostra; $\mathrm{P}=$ proporção estimada na população; $\mathrm{p}=$ proporção total; $\mathrm{e}_{\mathrm{a}}=$ erro amostral.

Objetivando estimar o tamanho amostral, usou-se a proporção estimada de 0,5 , uma vez que não existem estudos nacionais que informem a prevalência de utilização de equipamentos de tecnologia assistiva por idosos em uma comunidade, considerando um erro amostral de $10 \%$ e um nível de significância de 5\%. Assim, definiu-se uma amostra de 144 idosos.

De forma a garantir a abrangência da amostra de maneira representativa em todo o território pesquisado, isto é, nas nove microáreas, as 144 pessoas foram distribuídas entre elas, totalizando 16 indivíduos sorteados em cada uma.

Para a identificação das pessoas a serem entrevistadas, os 513 idosos foram numerados de forma ascendente e sorteados aleatoriamente 144 indivíduos, conforme o cálculo amostral. Nos casos elencados nos critérios de exclusão, a amostra foi recomposta por meio de novo sorteio aleatório. Quando em uma microárea todos os idosos já haviam sido sorteados, recorreu-se à outra de forma a manter o tamanho da amostra.

Para a coleta dos dados foi constituída uma equipe de cinco entrevistadores, quatro discentes e um docente, membros do Laboratório Integrado de Tecnologia Assistiva da Universidade Federal do Triângulo Mineiro (LITA/UFTM), treinados para aplicação dos instrumentos e para a forma de abordagem aos idosos e questões éticas da pesquisa. A coleta de dados ocorreu no domicílio entre os meses de agosto de 2014 a janeiro de 2015.

O questionário de pesquisa era constituído de duas seções: uma relacionada a questões sócio demográficas seguindo o "Critério de Classificação Econômica Brasil CCEB" da Associação Brasileira de Empresas de Pesquisa $(\mathrm{ABEP})^{10}$; e uma segunda, que incluiu a aplicação de formulário estruturado elaborado pelos pesquisadores autores contendo os aspectos pessoais (idade, estado civil e diagnóstico clínico) e questões fechadas sobre tecnologia assistiva com respostas binárias ( $\operatorname{sim} /$ não).

As questões relacionadas à classe econômica de acordo com a ABEP apontam a renda média familiar por classes como sendo A1 (R\$14.250,00), A2 (R\$7.557,00), $\mathrm{B} 1$ (R\$3.844,00), B2 (R \$ 2.256,00), C1 (R \$ 1.318,00), C2 ( $\mathrm{R} \$ 861,00), \mathrm{D}(\mathrm{R} \$ 573,00), \mathrm{E}(\mathrm{R} \$ 329,00)$.

As questões fechadas eram relacionadas à presença ou não de tecnologia assistiva na área de ocupação do participante envolvendo as atividades de vida diária, que segundo a Associação Americana de Terapia Ocupacional ${ }^{4}$ compreendem as atividades cotidianas de banho; alimentação; vestuário; mobilidade; uso do vaso sanitário; equipamentos pessoais; autocuidado e equipamentos de higiene pessoal.

$\mathrm{Na}$ atividade do 'banho' identificou-se o uso de cadeira ou banco para banho; tapete de borracha com ventosa; sabonete acoplado à bucha, fixado a um cordão ou luva atoalhada; esponja ou escova de cabo longo; barras de segurança e apoio. Para o 'vestuário' buscouse saber sobre o uso de abotoador, tênis e roupas com velcro, calçadeira, argola para puxador de zíper, sutiã com abertura frontal, gravata com elástico, órtese e prótese. $\mathrm{Na}$ 'alimentação': prato com bordas elevadas, prato com ventosa com superfície antiderrapante, prato com clipe de borda elevada, engrossador de cabo para alimentação, talheres com cabo adaptado (engrossado ou angulado), copo com tampa em bico ou com tampa e canudo, copo com alça unilateral ou bilateral, copo com recorte, copo com peso no fundo, bandeja para encaixe de prato e copo, correia universal para acoplar objetos. Já no 'cuidado com equipamentos pessoais' investigou-se sobre uso de órtese, prótese, aparelhos auditivos e óculos; enquanto que no item 'higiene pessoal e autocuidado': o engrossador de cabo para produtos de higiene pessoal e autocuidado, correia universal para acoplar objetos, órtese e prótese dentária. No item 'uso do vaso sanitário' foi investigado uso de assento sanitário elevado, elevação da altura do vaso sanitário (sóculo), cadeira de rodas higiênica, barras de segurança e apoio. No item 'mobilidade' foi perguntado sobre o uso de bengala, cadeira de rodas, muleta e/ou andador. 


\section{ANÁLISE DOS DADOS}

Os resultados coletados foram submetidos à análise descritiva por meio de frequência absoluta e relativa para as variáveis qualitativas (nível econômico, estado civil, gênero, diagnóstico clínico, tecnologia assistiva) e média e desvio padrão para a variável quantitativa (idade). Todas as análises foram realizadas no software Statistical Package for Social Sciences (SPSS, Chicago, IL, USA) versão 20.

\section{RESULTADOS}

As características sociodemográficas podem ser observadas na Tabela 1. A idade média dos participantes foi de 72,4 anos $( \pm 7,8)$, sendo a maior idade 92 anos e a menor 60 anos, e predomínio de idosos do gênero feminino (68\%). Em relação ao estado civil, predominam os casados $(57,6 \%)$ e a maioria dos pesquisados $(34,7 \%)$ compõem a classe econômica nível $\mathrm{C} 2$, que equivale a uma renda média familiar de $\mathrm{R} \$ 861,00$ (Tabela 1).

Tabela 1 - Características sociodemográficas dos participantes $(n=144)$

\begin{tabular}{l|c|c}
\hline Variável & $\boldsymbol{n}$ & $\mathbf{\%}$ \\
\hline Faixa Etária & & \\
\hline $60-69$ & 64 & 44,4 \\
$70-79$ & 52 & 36,1 \\
$80-89$ & 24 & 16,7 \\
$90-99$ & 04 & 02,8 \\
\hline Estado Civil & & \\
\hline Casado & 83 & 57,6 \\
Viúvo & 42 & 29,2 \\
Divorciado & 09 & 06,3 \\
Solteiro & 10 & 06,9 \\
\hline Gênero & & \\
\hline Feminino & 98 & 68,1 \\
Masculino & 46 & 31,9 \\
\hline Nível Econômico & & \\
\hline A1 & 00 & 00,0 \\
A2 & 01 & 00,7 \\
B1 & 00 & 00,0 \\
B2 & 24 & 16,6 \\
C1 & 37 & 25,7 \\
C2 & 51 & 35,4 \\
D & 26 & 03,5 \\
E & 05 & \\
\hline
\end{tabular}

Os diagnósticos clínicos relatados foram na maioria casos de hipertensão arterial $(42,3 \%)$, seguido por diabetes mellitus $(14,6 \%)$ e comorbidades associadas como diabetes mellitus e hipertensão arterial (10,3\%) (Tabela 2).

Tabela 2 - Diagnóstico clínico relatado pelos participantes $(n=144)$

\begin{tabular}{l|c|c}
\hline Diagnóstico & $\boldsymbol{n}$ & $\mathbf{\%}$ \\
\hline Acidente Vascular Encefálico & 06 & 04,2 \\
\hline Arritmia & 01 & 00,7 \\
\hline Condição Ortopédica - coluna & 03 & 02,1 \\
\hline Depressão & 01 & 00,7 \\
\hline Doença de Alzheimer & 02 & 01,4 \\
\hline Doença de Chagas & 04 & 02,8 \\
\hline Deficiência visual & 01 & 00,7 \\
\hline Diabetes & 21 & 14,6 \\
\hline Enfisema pulmonar & 02 & 01,4 \\
\hline Gota & 01 & 00,7 \\
\hline Hipertensão Arterial & 61 & 42,3 \\
\hline Hipertensão e Diabetes & 15 & 10,3 \\
\hline Labirintite & 04 & 02,8 \\
\hline Parkinson & 01 & 00,7 \\
\hline Sem diagnóstico & 21 & 14,6 \\
\hline
\end{tabular}

Com relação à presença de recursos de tecnologia assistiva (TA) em alguma atividade de vida diária, verificou-se que $140(97,2 \%)$ participantes relataram utilizar algum tipo de recurso de TA como auxilio em suas tarefas. Na Tabela 3 podem ser observadas as categorias investigadas na área de ocupação referida. Especificamente, no item 'banho, tomar banho', todos os cinco recursos pesquisados foram relatados por no mínimo $4 \%$ dos idosos participantes.

No vestuário, foram identificados oito recursos, mas apenas dois participantes $(1,4 \%)$ relataram utilizar TA, em específico, sutiã com abertura frontal devido à dificuldade de movimentos dos membros superiores e órtese. Porém, durante a coleta, foi referido por 16 $(11,1 \%)$ participantes a necessidade de auxílio de uma terceira pessoa para calçar meias e sapatos, assim como para fechar botões e zíperes.

$\mathrm{Na}$ alimentação havia doze recursos investigados. Destes, apenas dois foram apontados como sendo utilizados em atividades cotidianas. E, com relação aos cuidados com equipamentos pessoais, pode-se observar que 112 idosos fazem uso de um dos dois recursos investigados (Tabela 3 ). 
Tabela 3 - Recurso de tecnologia assistiva por categorias na área de ocupação - atividade de vida diária $(n=144)$

\begin{tabular}{|c|c|c|}
\hline Banho, tomar banho & $\mathbf{n}$ & $\%$ \\
\hline Banco ou cadeira para banho & 12 & 08,3 \\
\hline Tapete de borracha com ventosa & 62 & 43,1 \\
\hline Sabonete acoplado à bucha, fixado a um cordão & & \\
\hline ou luva atoalhada & 06 & 04,2 \\
\hline Esponja ou escova de cabo longo & 47 & 32,6 \\
\hline Barra de segurança e apoio & 17 & 11,8 \\
\hline Vestir-se & $\mathbf{n}$ & $\%$ \\
\hline Abotoadeira & 00 & 00,0 \\
\hline Tênis e roupas com velcro & 00 & 00,0 \\
\hline Calçadeira & 00 & 00,0 \\
\hline Argola para puxador de zíper & 00 & 00,0 \\
\hline Sutiã abertura frontal & 01 & 00,7 \\
\hline Gravata com elástico & 00 & 00,0 \\
\hline Órtese & 01 & 00,7 \\
\hline Prótese & 00 & 00,0 \\
\hline Alimentação & $\mathbf{n}$ & $\%$ \\
\hline Prato com bordas elevadas & 00 & 00,0 \\
\hline $\begin{array}{l}\text { Prato com ventosa com superfície } \\
\text { antiderrapante }\end{array}$ & 01 & 00,7 \\
\hline Prato com clipe de borda elevada & 00 & 00,0 \\
\hline $\begin{array}{l}\text { Talheres com cabo adaptado (engrossado ou } \\
\text { angulado) }\end{array}$ & 00 & 00,0 \\
\hline Copo com tampa em bico & 00 & 00,0 \\
\hline Copo com tampa e canudo & 00 & 00,0 \\
\hline Copo com alça unilateral & 06 & 04,2 \\
\hline Copo com alça bilateral & 00 & 00,0 \\
\hline Copo com recorte & 00 & 00,0 \\
\hline Copo com peso fundo & 00 & 00,0 \\
\hline Bandeja para encaixe de prato e copo & 00 & 00,0 \\
\hline Correia universal para acoplar objetos & 00 & 00,0 \\
\hline Cuidado com equipamentos pessoais & $\mathbf{n}$ & $\%$ \\
\hline Aparelho Auditivo & 08 & 05,5 \\
\hline Óculos & 104 & 72,2 \\
\hline Higiene pessoal e autocuidado & $\mathbf{n}$ & $\%$ \\
\hline $\begin{array}{l}\text { Engrossador de cabo para produtos de higiene } \\
\text { pessoal e autocuidado }\end{array}$ & 00 & 00,0 \\
\hline Correia universal para acoplar objetos pessoais & 00 & 00,0 \\
\hline Órtese dentária & 73 & 50,7 \\
\hline Prótese dentária & 58 & 40,3 \\
\hline Uso do vaso sanitário & $\mathbf{n}$ & $\%$ \\
\hline Elevação do assento do vaso sanitário & 04 & 02,8 \\
\hline Aumento da altura do vaso sanitário & 03 & 02,1 \\
\hline Barra de segurança e apoio & 09 & 06,3 \\
\hline Mobilidade & $\mathbf{n}$ & $\%$ \\
\hline Bengala & 18 & 12,5 \\
\hline Andador & 06 & 04,2 \\
\hline Muleta & 00 & 00,0 \\
\hline Cadeira de rodas & 05 & 03,5 \\
\hline
\end{tabular}

$\mathrm{Na}$ atividade de 'higiene pessoal e autocuidado', observou-se que os idosos relataram usar órteses dentárias e próteses dentárias. Quanto ao 'uso do vaso sanitário', os três recursos de TA pesquisados foram relatados por uma minoria dos idosos participantes. No item 'mobilidade', verificou-se uma preferência pela bengala.

\section{DISCUSSÃO}

Nos resultados encontrados foi observado que $42,3 \%$ dos idosos entrevistados relataram hipertensão arterial. Esse evento pode ser explicado pelas alterações fisiológicas resultantes do processo de envelhecimento, como o aumento da rigidez das artérias, ocasionando redução da distensibilidade vascular e por consequência, aumento da pressão arterial $^{11}$. Cabe ressaltar que a prevalência encontrada é menor que o apontado por estudos populacionais realizados em outros estados com população na mesma faixa etária ${ }^{12,13}$. Para Alves et al. ${ }^{14}$ a hipertensão é uma doença crônica que pode aumentar em 39\% a dependência na realização das atividades instrumentais de vida diária.

A maioria dos participantes possuía renda média familiar de R $\$ 861,00$, valor pouco superior ao salário mínimo ${ }^{15}$. Independente da prevalência encontrada cabe ressaltar que segundo Pinto e Neri ${ }^{16}$ saúde e capacidade funcional são integradas à "renda familiar, à escolaridade, à idade e ao sexo. No Brasil, os efeitos desses fatores associam-se a dificuldades de acesso a serviços de saúde e à escassez de informações e de oportunidades para a manutenção das condições físicas e funcionais na velhice" (p.3455) ${ }^{16}$.

Cruz e Emmel ${ }^{2}$, Nunes et al. ${ }^{11}$ e Giacomin et al. ${ }^{5}$, relatam que a perda da capacidade funcional é um fator relevante no decorrer do ciclo da vida, já que ocorre a diminuição gradativa das habilidades e consequentemente das capacidades para desempenhar atividades do dia a dia. Uma medida preventiva, disponível como auxílio para a manutenção da capacidade, é o uso de tecnologia assistiva ${ }^{17}$. Que se contrapõe aos resultados obtidos neste trabalho, pois embora tenha sido apontado que 97,2\% dos participantes utilizam qualquer equipamento de assistência descrito na ocupação pesquisada, a quantidade e o tipo de tecnologia assistiva em cada atividade que o idoso se envolve são restritos e limitados. Isso implicaria em limitações funcionais mesmo que o indivíduo faça uso de tecnologia assistiva, pois os equipamentos usados são restritos a um determinado grupo que não cobre toda a área de ocupação investigada. Esses dados traduzem uma situação paradigmática em que o uso de tecnologia 
assistiva é alto (97,2\%), mas mesmo assim o potencial funcional dos sujeitos não é aproveitado. Considerando isso, uma avaliação da necessidade do idoso, seguida de seleção do recurso e treino para seu uso, isto é, a adequação na variedade de equipamentos prescritos e distribuídos aos idosos, poderia diminuir o déficit funcional que pode existir. A situação de contradição apresentada acima, em que se tem um alto uso de tecnologia assistiva que pode não se reproduzir em incremento ou manutenção da funcionalidade evidencia um descompasso entre as necessidades funcionais do idoso e a oferta de tecnologia assistiva pelo Sistema Único de Saúde (SUS).

Sobre o uso de tecnologia assistiva segundo a área de ocupação, em relação ao item 'banho, tomar banho', destaca-se que a maioria dos participantes, não possui dispositivos de TA para auxiliar seu desempenho nessa atividade. Alguns equipamentos como tapete antiderrapante e barra de apoio tem estreita relação com a segurança dos idosos e podem prevenir quedas. De acordo com Chianca et al. ${ }^{19}$, pessoas de qualquer faixa etária podem sofrer quedas, mas em pessoas idosas este risco é maior e as consequências são significativas para sua condição de saúde, pois poderá acarretar incapacidade, lesão e óbito. Para Cruz et al..$^{20}$ a idade avançada esta associada ao aumento do risco de quedas. A informação de baixa frequência de uso de tecnologia assistiva nesse contexto é importante, porque o uso preventivo desses equipamentos poderia influenciar na diminuição das quedas, e por consequência evitar a necessidade de uso de serviços de saúde por essa causa, levando também à economia pelo sistema de saúde, além da manutenção funcional do idoso. Portanto, adequações relacionadas aos equipamentos para uso no banho poderiam ser estimuladas na área estudada, por meio de ações dos profissionais da atenção básica, voltadas para avaliação, prescrição, seleção, confecção e treino de uso do recurso de tecnologia assistiva.

Os itens 'vestuário' e 'alimentação' apresentaram poucos relatos de uso de tecnologia assistiva. Sabe-se que o envelhecimento altera as habilidades físicas, cognitivas e pode trazer o agravo advindo das doenças e ocasionar dependência das atividades cotidianas ${ }^{21}$. De acordo com a Associação Americana de Terapia Ocupacional ${ }^{4}$, vestuário e alimentação são atividades rotineiras que $o$ indivíduo desempenha em seu dia a dia. Uma vez que o envelhecimento altera as funções físicas, os idosos podem ter dificuldades em executar essas tarefas. Uma hipótese para o baixo número de recursos de TA nesses itens seria a assistência constante de um cuidador ou membro da família na realização das tarefas ou a não dependência do idoso e, portanto a ausência da necessidade do recurso de tecnologia assistiva.

No 'cuidado com equipamentos pessoais' verificouse a maior prevalência de óculos para leitura. É sabido, que na fase do envelhecimento surgem queixas sobre a acuidade visual e auditiva, porém essas não atrapalham o envelhecer ativo, já que existem meios para diminuir as dificuldades inerentes ao processo natural que compõe a idade avançada, como as órteses (óculos e aparelho auditivo), permitindo os ajustes dessas deficiências ${ }^{11}$.

Sobre o item 'higiene pessoal e autocuidado', a maioria dos idosos relatou possuirórtese ou prótese dentária. O que corrobora com o relato de Simões e Carvalho ${ }^{22}$, que explicam que a doença periodontal tende a aumentar com o avanço da idade e tem sido identificada como a causa primária de ausência de dente conduzindo para um número alto de pessoas que demandam protetização.

No item 'mobilidade', não foi relatado frequência de uso de equipamentos de auxílio para a marcha pelos participantes; no entanto, pode ser percebido durante a coleta de dados que alguns idosos necessitavam deste tipo de tecnologia, contudo não os tinham. Cruz et al. ${ }^{20}$ apontam que $11 \%$ dos idosos estudados em uma determinada região demandavam auxílio para o deslocamento, seja ele por parte de um cuidador ou pelo uso regular de um dispositivo de auxílio para marcha. Chianca et al. ${ }^{19}$ esclarecem que a idade de uma pessoa acima de 65 anos é um fator predisponente para quedas, destacando que $81,3 \%$ dos participantes do estudo estavam incluídos nessa probabilidade. Esse é um componente funcional que merece especial atenção, pois a literatura aponta relação entre a capacidade de deslocamento e a funcionalidade ${ }^{23,24,25}$, por isso, o descolamento deve ser estimulado para que o decréscimo da funcionalidade tenha menor impacto.

A tecnologia assistiva (TA) constitui opção enquanto serviço dispensado pelo SUS visando oferecer recursos, produtos e estratégias a fim de solucionar as demandas advindas dos agravos do envelhecimento, como a mobilidade reduzida, a alteração da capacidade funcional e aumento da dependência ${ }^{6}$. Os dispositivos de TA buscam auxiliar o indivíduo no envolvimento em qualquer área de ocupação, como nas atividades de vida diária (banho, alimentação e vestuário, por exemplo) proporcionando melhor desempenho funcional ${ }^{4}$.

Entre as limitações dessa pesquisa pode-se citar a restrição do estudo a uma área geográfica da cidade, acarretando a não representatividade dos dados. Além disso, a real necessidade da TA entre idosos não foi avaliada, sendo coletadas somente informações a respeito de seu uso. A funcionalidade dos idosos mesmo 
com o uso dos equipamentos poderia ter sido avaliada, assim como a prescrição, origem e uso adequado dos equipamentos. Entretanto, cabe ressaltar que este estudo apresentou uma descrição detalhada dos equipamentos em uso por idosos em uma região da cidade de Uberaba, MG. Além disso, o processo amostral criteriosamente conduzido garante validade interna e qualidade aos resultados encontrados.

\section{CONCLUSÕES}

Conclui-se que na população estudada houve relato de uso de recursos de tecnologia assistiva em alguma atividade de vida diária. No entanto, quando se especificando os itens de equipamentos de assistência elencados em cada atividade de vida diária evidenciou-se uma utilização aquém para o desempenho funcional dos idosos, com exceção do uso de óculos.

O envelhecimento é uma fase que pode ocasionar disfunções motoras, diminuição da capacidade funcional e dependência, mas os idosos apesar de apresentarem essas características nessa fase da vida, podem ter

\section{REFERÊNCIAS}

1. Araújo DD, Azevedo RS, Chianca TCM. Perfil demográfico da população idosa de Montes Claros, Minas Gerais e Brasil. RECOM Rev Enferm Cent O Min. 2011;1(4):4629. Disponível em: http://www.seer.ufsj.edu.br/index.php/ recom/article/view/151/236.

2. Cruz DMC DA, Emmel MLG. Papéis ocupacionais de pessoas com deficiências físicas: diferenças de gênero e ciclos de desenvolvimento. Rev Baiana Ter Ocup (Salvador). 2012;1(1):4-24. Disponível em: https://www5.bahiana.edu. br/index.php/terapiaocupacional/article/view/124.

3. Brasil. Ministério da Saúde. Secretaria de Atenção à Saúde. Departamento de Atenção Básica. Envelhecimento e saúde da pessoa idosa. Brasília; 2007. Disponível em: http:// bvsms.saude.gov.br/bvs/publicacoes/abcad19.pdf.

4. Associação Americana de Terapia Ocupacional (AOTA). Occupational Therapy Practice: framework: domain \& process: 3rd. ed. Am J Occup Ther. 2014;68(Suppl 1):S1-48. Available from: http://ajot.aota.org/article. aspx?articleid=1860439.

5. Giacomin KC, Peixoto SV, UchoaE, Costa MFL. Estudo de base populacional dos fatores associados à incapacidade funcional um envelhecimento ativo, saudável e que pode ser potencializado com a utilização de TA. Assim, uma sugestão para novo estudo é a investigação para além da variedade de equipamentos, ou seja, quais as variáveis individuais, ambientais e culturais que influenciam o uso de TA por idosos. Assim como se sugere que outros estudos continuem a investigar a temática incluindo pesquisa sobre a concordância dos dispositivos com as demandas de desempenho desses idosos; isto é, se a tecnologia assistiva está apropriada ou adequada.

A disponibilização dessas informações poderá resultar em melhorias na qualidade dos serviços na rede pública e por consequência, também incrementos na qualidade de vida da população idosa em uso de tecnologia assistiva. Além do que, já foi citado poderá trazer contribuições ao campo da pesquisa, auxiliando a sedimentação e agregação de conhecimento na medida em que traz novas informações, contribuindo para a expansão do campo de TA por idosos. Ao Sistema de Saúde, este estudo oferece dados que podem subsidiar a tomada de decisão, por meio de políticas ou programas de cuidado e assistência ao grupo populacional estudado. entre idosos na Região Metropolitana de Belo Horizonte, Minas Gerais, Brasil. Cad Saúde Pública. 2008;24(6):1260-70. http://dx.doi.org/10.1590/S0102-311X2008000600007.

6. Brasil. Ministério da Saúde. Portal da Saúde. Portaria $n^{\circ}$ 3.089, de 11 de dezembro de 2013. Brasília; 2014 [citado em 26 set. 2014]. Disponível em: http://bvsms.saude.gov. br/bvs/saudelegis/gm/2013/prt3089_11_12_2013.html.

7. Brasil. Subsecretaria Nacional de Promoção dos Direitos da Pessoa com Deficiência. Comitê de Ajudas Técnicas. Tecnologia Assistiva. Brasília: CORDE; 2009. Disponível em: http://www.pessoacomdeficiencia.gov.br/app/sites/default/ files/publicacoes/livro-tecnologia-assistiva.pdf.

8. Brasil. Ministério da Saúde. Portal da Saúde. Anexo portaria $\mathrm{n}^{\mathrm{o}} 3.089$, de 11 de dezembro de 2013 lista de produtos estratégicos para o SUS Brasília; 2014 [citado em 26 set. 2014]. Disponível em: http://portalsaude.saude.gov.br/ images/pdf/2013/dezembro/23/anexo-portaria3089.pdf.

9. Garcia JCD, Galvão Filho TA. Pesquisa nacional de tecnologia assistiva. São Paulo: ITS Brasil;2012. Disponível em: http://www.itsbrasil.org.br/sites/itsbrasil.w20.com.br/ files/Digite $\% 20$ o $\% 20$ texto/miolopesqnacional-grafica.pdf. 
10. ABEP. Associação Brasileira das Empresas de Pesquisa. Critério de Classificação Econômica Brasil [citado em 11 ago. 2014]. Disponível em: file:///C:/Users/Administrador/ Downloads/07_cceb_2008_em_vigor_em_2008_base 1se_2005.pdf.

11. Nunes DP, Nakatani AYK, Silveira EA, Bachion MM, Souza MR. Capacidade funcional, condições socioeconômicas e de saúde de idosos atendidos por equipes de Saúde da Família de Goiânia (GO, Brasil). Cienc Saúde Coletiva. 2010;15(6):288798. http://dx.doi.org/10.1590/S1413-81232010000600026.

12. Zaitune MPA, Barros MBA, César CLG, Carandina L, Goldbaum M. Hipertensão arterial em idosos: prevalência, fatores associados e práticas de controle no Município de Campinas, São Paulo, Brasil. Cad Saúde Pública. 2006;22(2):285-94. https://dx.doi.org/10.1590/ S0102-311X2006000200006.

13. Esperandio EM, Espinosa MM, Martins MAS, Guimarães LV, Lopes MAL, Nazário Scala LCN. Prevalência e fatores associados à hipertensão arterial em idosos de municípios da Amazônia Legal, MT. Rev Bras Geriatr Gerontol. 2013;16(3):481-93. http://dx.doi.org/10.1590/ S1809-98232013000300007.

14. Alves LC, Leiman BCQ, Vasconcelos MEL, Carvalho MS, Vasconcelos AGG, Fonseca TCO, Lebrão ML, Laurenti R. A influência das doenças crônicas na capacidade funcional dos idosos. Cad Saúde Pública. 2007;23(8):1924-30. http:// dx.doi.org/10.1590/S0102-311X2007000800019.

15. Brasil. Poder Executivo - decreto n 8.381 de 29.12.2014 [citado em 12 abr. 2015]. Disponível em: http://www. normaslegais.com.br/legislacao/Decreto-8381-2014.htm.

16. Pinto JM, Neri AL. Doenças crônicas, capacidade funcional, envolvimento social e satisfação em idosos comunitários: estudo fibra. Ciênc Saúde Coletiva. 2013;18(12):3449-60. http://dx.doi.org/10.1590/S1413-81232013001200002.

17. Bain BK. Tecnologia de assistência na terapia ocupacional. In: Neistadt ME, Crepeau EB. Willard \& Spackman: terapia ocupacional. 9a ed. Rio de Janeiro: Guanabara Koogan; 2002. p.463-79.

18. Viana ALA, Fausto MCR, Lima LD. Política de saúde e eqüidade. São Paulo Perspectiva. 2003; 17(1):58-68. doi: 10.1590/S0102-88392003000100007.

19. Chianca TCM, Andrade CR, Albuquerque J, Wenceslau LCC, Tadeu LFR, Macieira TGR, Ercole FF. Prevalência de quedas em idosos cadastrados em um Centro de Saúde de Belo Horizonte-MG. Rev Bras Enferm (Brasília). 2013;66(2):23440. http://dx.doi.org/10.1590/S0034-71672013000200013.

20. Cruz DT, Ribeiro LC, Vieira MT, Teixeira MTB, Bastos RR, Leite ICG. Prevalência de quedas e fatores associados em idosos. Rev Saúde Pública. 2012;46(1):138-46. http:// dx.doi.org/10.1590/S0034-89102007000500009.

21. Fiedler MM, Peres KG. Capacidade funcional e fatores associados em idosos do Sul do Brasil: um estudo de base populacional. Cad Saúde Pública. 2008;24 (2):409-15. http://dx.doi.org/10.1590/S0102-311X2008000200020.

22. Simões ACA, Carvalho DM. A realidade da saúde bucal do idoso no Sudeste brasileiro. Ciênc Saúde Coletiva. 2011;16(6):2975-82. http://dx.doi.org/10.1590/S141381232011000600035 .

23. Scherder E, Scherder R, Verburgh L, Königs M, Blom M, Kramer AF, Eggermont L. Executive functions of sedentary elderly may benefit from walking: a systematic review and meta-analysis. Am J Geriatr Psychiatry. 2014;22(8):782-91. doi: 10.1016/j.jagp.2012.12.026.

24. Heiwe S, Tollbäck A, Clyne N. Twelve weeks of exercise training increases muscle function and walking capacity in elderly predialysis patients and healthy subjects. Nephron. 2001;88(1):48-56. doi: 10.1159/000045959.

25. Shin, Y. The effects of a walking exercise program on physical function and emotional state of elderly Korean women. Public Health Nurs. Malden. 1999; 16 (2):146-54. doi: 10.1046/j.1525-1446.1999.00146.x. 